\title{
Éditorial
}

\section{Nous sommes tous Japonais}

Le fait majeur de ces dernières semaines est sans conteste le tremblement de terre qui a ravagé une partie du Japon, suivi d'un tsunami tout aussi dévastateur. Ensuite vinrent les conséquences sur les réacteurs de la centrale de Fukushima.

Nos premières pensées sont allées à nos amis japonais et tout particulièrement à notre correspondant au Japon, Endo Akira. Le Comité de rédaction a été particulièrement ému par son courriel où il nous disait combien la vie est difficile lorsqu'on a tout perdu, que l'eau, le gaz, l'électricité et la nourriture vous manquent, mais il nous a donné une leçon de dignité et de courage en nous rassurant car lui et sa famille avaient survécu.

Merci Endo Akira pour cette magnifique leçon d'humanité.

Bien entendu il faut parler des conséquences en terme de radioprotection des accidents eux mêmes. Les experts de la sûreté en tireront sûrement toutes les leçons, et ils vont avoir du travail. Pour nous, experts en radioprotection, nous pouvons dire que toutes les leçons tirées de la catastrophe de Tchernobyl ont été fort utiles à nos amis japonais ; ils ont distribué à temps les pastilles d'iode, évitant ainsi l'apparition massive de cancers de la thyroïde, ils ont évacués raisonnablement, évitant là encore des conséquences dramatiques pour les populations.

Malgré quelques critiques, la communication a bien marché, nous avons là encore tiré les leçons des exercices INEX réalisés par l'AEN.

Dans quelques mois, nous aurons une évaluation plus précise des zones contaminées qu'il faudra reconquérir, le programme européen «post Tchernobyl » ETHOS leur sera fort utile.

Enfin n'oublions pas que RADIOPROTECTION a consacré un numéro spécial au programme européen EURANOS qui traite des situations post accidentelles. N'oublions pas également l'immense travail fait en France dans le cadre du CODIRPA.

Oui, toutes les leçons que nous pouvions tirer de l'accident de Tchernobyl ont été notre actualité ces dernières années, Fukushima nous montre que ce n'était pas du temps perdu.

Henri Métivier 